\title{
Mediciones de Estrés Laboral en Docentes de un Colegio Público Regional Chileno
}

\author{
Sergio Zuniga-Jara* y Victor Pizarro-Leon \\ Universidad Católica del Norte, Escuela de Ciencias Empresariales, Larrondo 1281, Coquimbo-Chile \\ (e-mail: sz@ucn.cl; victor.pizarroleon@live.com)
}

${ }^{*}$ Autor a quien debe ser enviada la correspondencia

Recibido Jun. 2, 2017; Aceptado Ago. 16, 2017; Versión final Sep. 28, 2017, Publicado Feb. 2018

\begin{abstract}
Resumen
Esta investigación analiza la existencia del síndrome de Burnout en docentes de un colegio público de enseñanza técnico profesional de la ciudad de Coquimbo, en Chile. La recolección de datos se realizó a través del cuestionario Maslach Burnout Inventory (MBI). Como resultado, el nivel de Síndrome de Burnout global es de tipo medio o bajo en los educadores del colegio. Solamente el agotamiento emocional mostró una incidencia importante ( $34 \%$ de los educadores encuestados), mientras que los problemas de despersonalización y de falta de realización personal en el trabajo se encuentran en niveles bajos y bajomedio respectivamente.
\end{abstract}

Palabras clave: síndrome de burnout; Maslach burnout inventory; burnout; colegio público

\section{Measurements of Labor Stress in Teachers of a Regional Public High School}

\begin{abstract}
This research analyzes the existence of Burnout syndrome in teachers of a public high school of technical education in the city of Coquimbo, in Chile. Data collection was done using the Maslach Burnout Inventory (MBI). As a result, the global level of Burnout Syndrome is medium or low in the educators of this public high school. Only emotional exhaustion showed significant incidence (34\% of the surveyed educators), while the problems of depersonalization and lack of personal accomplishment at work are found at low and lowmedium levels respectively.
\end{abstract}

Keywords: burnout syndrome; Maslach burnout inventory; burnout; public high school 


\section{INTRODUCCIÓN}

El estrés laboral puede incidir negativamente en el desempeño de los docentes de un establecimiento educacional, lo que ha dado origen a diversos estudios relacionados con la salud física y mental de los profesores. Especialmente los docentes de los establecimientos educacionales del sector público pueden enfrentar un excesivo número de estudiantes en aula, indisciplina, falta de interés por aprender y materiales de trabajo inadecuados; y entonces estos docentes podrían estar más expuestos a altos índices de estrés laboral. Esto podría significar una menor capacidad para generar procesos de aprendizaje profundos en el alumnado, y en el caso de directivos podría afectar su habilidad de gestionar eficientemente los recursos del establecimiento. Todo esto puede traer como consecuencia bajos puntajes en el Sistema de Medición de la Calidad de la Educación (SIMCE) y la Prueba de Selección Universitaria (PSU), afectando el prestigio de la institución, generando una baja en el número de matrículas.

El estrés laboral es una respuesta emocional, fisiológica y conductual, potencialmente patógena, que influye en la actividad laboral de los docentes, y en su resultado pedagógico. Esto lleva a una pérdida de motivación, la que suele progresar hacia sentimientos de inadecuación y fracaso (Lazarus y Folkman, 1984), un deterioro en la salud y en las relaciones interpersonales, dentro y fuera del ámbito laboral. Por ello es importante detectar los síntomas de estrés laboral en docentes. A partir de los resultados de mediciones apropiadas es posible pensar en un programa orientado a fortalecer el ambiente académico y el clima organizacional de la institución, permitiendo crear, mantener y mejorar las condiciones de trabajo, y mejorando la calidad de vida de las familias. Además, se pueden esperar aumentos en los niveles de satisfacción e identificación con el establecimiento en el cual se trabaja, beneficiando a toda la comunidad educativa.

La respuesta psicológica de un profesional al estrés laboral crónico se conoce como el Síndrome de Burnout ("trabajador quemado") (Maslach, 1976 y Maslach y Jackson, 1986). El Síndrome de Burnout fue descrito en el año 1969, denominado en sus principios como 'Staff Burnout', para referirse al extraño comportamiento que presentaban algunos oficiales de policías. Después de dos décadas, este síndrome no ha tenido una definición totalmente aceptada por psicólogos e investigadores, debido a que algunos lo consideran estrés, y otros lo denominan cansancio emocional (Craiovan, 2014). La definición más aceptada indica que este síndrome se manifiesta en tres síntomas: cansancio emocional (CE), despersonalización (DP) en el trato con sus clientes o usuarios, y la dificultad para la realización personal (RP). El Síndrome de Burnout es generalmente medido en tres dimensiones: a) agotamiento emocional, b) despersonalización, y c) falta de realización personal en el trabajo. A partir de estos tres componentes es usual basarse en el "Maslach Burnout Inventory" (MBI) por medio de tres subescalas de medición.

A nivel internacional, existe un importante cuerpo de investigaciones que analiza la incidencia de este síndrome en académicos, aunque también es relevante en otras áreas (Báez, et al., 2013). Mérida-López y Extremera (2017) proporcionan una amplia revisión de las principales investigaciones de como variables de personalidad están relacionadas con el estrés y burnout en profesores. Lauermann y König (2016) reportan que la competencia profesional de los profesores, es decir su conocimiento profesional, habilidades, creencias y motivación, son un factor de predicción crítico del bienestar y éxito profesional. Este conocimiento pedagógico general predice negativamente el burnout del maestro, tanto directa como indirectamente, a través de su asociación positiva con la autoeficacia de la enseñanza.

Fiorilli et al. (2015) proporcionan más evidencia del papel protector que ejerce el apoyo social en los profesores. Kim et al. (2017) encuentran que la exposición organizacional tuvo la asociación fuerte con el burnout de los profesores principiantes, y la importancia de la exposición a redes de apoyo social. Košir et al. (2015) examinaron el papel de la atención centrada en los síntomas de la angustia y en sus posibles causas y consecuencias, y no en sus soluciones (ruminación) en la predicción del estrés y el burnout de los profesores. Encontraron que la ruminación fue un predictor positivo directo del estrés y el burnout. Wang et al. (2015) analizaron si una mayor autoeficacia del maestro logra motivar a los estudiantes mejor, encontrando evidencia positiva, de modo que programas dirigidos a la autoeficacia del profesor pueden ser altamente efectivos. Lim y Eo (2014) encontraron que los diálogos reflexivos entre profesores se relacionaron negativamente con el nivel de burnout de los profesores, de tal modo que la política organizacional dentro de las escuelas está positivamente relacionada con el burnout. Van den Berghe et al. (2014) examinaron las diferencias en la satisfacción de necesidades, la necesidad de apoyo y el burnout entre los profesores, encontrando que el grupo con motivación de buena calidad informó más satisfacción de sus necesidades y apoyo que el grupo con motivación de baja calidad. La relación entre el burnout y la oportunidad de aprender y enseñar forman un campo de desarrollo que también ha sido explorado por varios autores (Panari et al., 2010, y Cárdenas y Hernández, 2011).

En Latinoamérica, Colino y Pérez (2015) estudian la incidencia del síndrome en profesores de centros de enseñanza públicos en Montevideo, encontrando que el 4,2\% de los docentes presentaron elevados niveles 
del síndrome, y un $42 \%$ altos valores en al menos una de sus dimensiones. Correa-Correa (2012) analizan el burnout en profesores universitarios de sectores público y privado de México, encontrando sólo en el sector público, aunque en un mínimo porcentaje, que los profesores presentaron antecedentes del síndrome con consecuencias psicológicas. Mosquera et al. (2016) predicen el grado de riesgo psicosocial en docentes de colegios colombianos.

En Chile, los estudios de este síndrome son escasos para el caso de profesores, predominando aquellos realizados en el sector salud. Por ejemplo, Muñoz y Molina (2013) analizan la Unidad de Cuidados Intensivos de un Hospital de Concepción, encontrando una baja presencia del síndrome, y altos niveles de realización personal, además de un desarrollo personal y profesional adecuado en todos los estamentos estudiados. Werner (2010) entrevista a profesionales que trabajan en salud pública (Cosam en Santiago de Chile), encontrando fuentes relevantes de Burnout asociadas a un clima laboral conflictivo, y a las demandas de los pacientes. Muñoz (2008) analiza al equipo de trabajadores de un centro de salud familiar de Valdivia, encontrando que un $12 \%$ de los funcionarios están afectados por el Burnout, que el cansancio emocional alto es significativo, la realización profesional es baja y el nivel de despersonalización al igual que el cansancio emocional es importante. Olivares et al. (2014) estudia trabajadores chilenos de distintas actividades, incluyendo a profesores de educación primaria y secundaria, encontrando relaciones significativas y en la dirección esperada: agotamiento emocional respecto de realización personal en el trabajo y despersonalización, y realización personal en el trabajo con despersonalización.

En el caso de estudios específicos del síndrome en el sector educacional de Chile, la literatura es escasa. Puede citarse a Buzzetti (2005), quien se investiga la validez factorial del MBI en profesores dirigentes sindicales del Colegio de Profesores A.G., concluyendo que la solución bifactorial permitiría evaluar el síndrome con un buen nivel de validez y confiabilidad. Quaas (2006) estudia a docentes universitarios de la quinta región de Chile, estimando que el $72 \%$ no presenta burnout, mientras que del $28 \%$ restante, el 16,1\% presenta un nivel reducido, un 10,2\% presenta nivel moderado, y sólo el 1,5\% está en los niveles altos de burnout. Ortiz et al. (2012) analizan burnout en educadoras de párvulos, encontrando diferencias en el puntaje total de burnout entre los estilos de personalidad "espectador" y "escéptico", y en agotamiento emocional entre los estilos "escéptico" y "complicado".

A partir de lo planteado en los antecedentes, el objetivo de esta investigación es detectar la existencia del síndrome de Burnout (agotamiento emocional, despersonalización y falta de realización profesional) en docentes de un liceo público de enseñanza técnico profesional de la ciudad de Coquimbo, Región de Coquimbo. Esta selección obedeció, por una parte, a la facilidad de acceso del equipo de investigadores para reunirse con los docentes y directivos de este liceo, y por otro lado, pensado en que esta sea una aplicación inicial de la metodología, para en una segunda etapa considerar una muestra más amplia. La hipótesis principal de este estudio es que existen altos niveles de Burnout por agotamiento emocional, por despersonalización, y por falta de realización personal. Otras hipótesis evaluadas son que los niveles medios de Burnout son iguales para jóvenes y mayores.

El resto del trabajo se organiza como sigue: A continuación se presenta la metodología, luego los resultados, y se finaliza con discusión final y las conclusiones.

\section{METODOLOGÍA}

Para la detección del Burnout se utilizó el cuestionario de Maslach Burnout Inventory (MBI) elaborado por Maslach y Jackson (1986), el cual es el instrumento más utilizado para la evaluación de este síndrome. El $\mathrm{MBI}$ es un instrumento que ha mostrado poseer una alta consistencia y fiabilidad, cercana al $90 \%$. Se anexó una encuesta para recolectar información de la edad, y otros antecedentes socioeconómicos de los docentes.

\section{El Inventario de Maslach para Agotamiento (MBI)}

EI MBI está constituido por 22 preguntas en forma de afirmaciones sobre los sentimientos y actitudes del profesorado en su trabajo diario con los estudiantes. Cada pregunta tiene asociada una escala de 0 a 6 , como sigue: 0 = Nunca, 1 = Alguna vez al año o menos, 2 = Una vez al mes o menos, 3 = Algunas veces al mes, 4 = Una vez a la semana, 5 = Varias veces a la semana, y $6=$ A diario. El cuestionario está dividido en tres subescalas: a) Agotamiento emocional; b) Despersonalización; y c) Realización personal.

a) Agotamiento emocional (EE: Emotional Exhaustion): 9 preguntas que valoran si se está exhausto emocionalmente por las demandas de trabajo. Puntuación máxima 9x6=54.

b) Despersonalización (D): 5 ítems que valoran el grado de frialdad y distanciamiento del encuestado. Puntuación máxima es $5 \times 6=30$. 
c) Realización personal (PA: Personal Accomplishment): 8 preguntas que evalúan los sentimientos de autoeficacia y realización personal en el trabajo. Las preguntas en este caso están redactadas en sentido contrario a las anteriores, de modo que los puntajes de cada pregunta deben ser invertidos, como sigue: Puntaje final $=6$ - Puntaje inicial. Puntuación máxima es $8 \times 6=48$.

La Tabla 1 muestra los rangos de valores que permiten identificar si el nivel de Burnout es bajo, medio o alto. Las variables de la base de datos se muestran en la Tabla 2.

Tabla 1: Rangos de Valores de Referencia para el IMB en cada dimensión

\begin{tabular}{lccc}
\hline & Bajo Burnout & Medio Burnout & Alto Burnout \\
\hline Agotamiento emocional & $0-18$ & $19-26$ & $27-54$ \\
Despersonalización & $0-5$ & $6-9$ & $10-30$ \\
Realización personal & $0-7$ & $8-14$ & $15-48$ \\
\hline
\end{tabular}

Tabla 2: Variables que conforman la base de datos

\begin{tabular}{|c|c|}
\hline Variable & Descripción \\
\hline Id & Número correlativo \\
\hline Profesión & $0=$ No especificado, $3=$ Directivo, $6=$ Historia y geografía, $9=$ Lenguaje, etc \\
\hline Genero & $0=$ Mujer; $1=$ Hombre \\
\hline Edad & Edad del entrevistado en años \\
\hline Formación & $0=$ No tiene; 1 =curso $/$ diplomado; $2=$ pos título; $3=$ magister \\
\hline Experiencia & Años de experiencia en docencia \\
\hline Contrato & $1=$ contrata; $2=$ indefinido \\
\hline Horas & Horas de docencia en aula semanal \\
\hline \multicolumn{2}{|c|}{$\begin{array}{l}\text { Las siguientes } 22 \text { preguntas tienen } 6 \text { opciones de respuesta: } \\
0: \text { Nunca; } 1 \text { : Alguna vez al año o menos; } 2 \text { : Una vez al mes o menos; } 3: \text { Algunas veces al mes; } \\
\text { 4: Una vez a la semana; } 5: \text { Varias veces a la semana } 6: \text { A diario }\end{array}$} \\
\hline & 1 Subescala: Agotamiento emocional \\
\hline b01 & Debido a mi trabajo me siento emocionalmente agotado. \\
\hline b02 & Al final de la jornada me siento agotado. \\
\hline b03 & $\begin{array}{l}\text { Me encuentro cansado cuando me levanto por las mañanas y tengo que enfrentarme a otro día } \\
\text { de trabajo. }\end{array}$ \\
\hline b06 & Trabajar con alumnos todos los días es una tensión para mí. \\
\hline b08 & Me siento "quemado" por el trabajo. \\
\hline b13 & Me siento frustrado por mi trabajo. \\
\hline b14 & Siento que estoy haciendo un trabajo demasiado duro. \\
\hline b16 & Trabajar en contacto directo con los alumnos me produce bastante estrés. \\
\hline \multirow[t]{2}{*}{ b20 } & En el trabajo siento que estoy al límite de mis posibilidades. \\
\hline & 2 Subescala: Despersonalización \\
\hline b05 & Creo que trato a algunos alumnos como si fueran objetos. \\
\hline b10 & Creo que tengo un comportamiento más insensible con la gente desde que hago este trabajo. \\
\hline b11 & Me preocupa que este trabajo me esté endureciendo emocionalmente. \\
\hline b15 & Realmente no me importa lo que les ocurrirá a algunos de mis alumnos. \\
\hline \multirow[t]{2}{*}{ b22 } & Siento que los alumnos me culpan de algunos de sus problemas. \\
\hline & 3 Subescala: Realización personal \\
\hline b04 & Puedo entender con facilidad lo que piensan mis alumnos. \\
\hline b07 & Me enfrento muy bien con los problemas que me presentan mis alumnos. \\
\hline b09 & Siento que mediante mi trabajo estoy influyendo positivamente en la vida de otros. \\
\hline b12 & Me encuentro con mucha vitalidad. \\
\hline b17 & Tengo facilidad para crear una atmósfera relajada en mis clases. \\
\hline b18 & Me encuentro animado después de trabajar junto con los alumnos. \\
\hline b19 & He realizado muchas cosas que merecen la pena en este trabajo. \\
\hline b21 & Siento que sé tratar de forma adecuada los problemas emocionales en el trabajo. \\
\hline
\end{tabular}


La unidad de análisis en este estudio son los docentes del liceo de enseñanza técnico profesional. Trabajan en el liceo 50 docentes y 7 directivos. El tamaño muestral mínimo (personas a encuestar) es:

$$
\mathrm{n}=\frac{\mathrm{N}^{*} \mathrm{Z}^{2 *} \mathrm{p}^{*}(1-\mathrm{p})}{(\mathrm{N}-1)^{*} \mathrm{e}^{2}+\mathrm{Z}^{2 *} \mathrm{p}^{*}(1-\mathrm{p})}=\frac{57 \times 1,65^{2} \times 50 \% \times(1-50 \%)}{(57-1) \times 0,10^{2}+1,65^{2} \times 50 \% \times(1-50 \%)}=38,80 / 1,24=31
$$

Donde:

$\mathrm{n}$ = tamaño mínimo de la muestra (número de encuestas).

$\mathrm{N}=$ tamaño del universo (57 educadores).

$Z=$ nivel de confianza $90 \%(Z=1,65)$.

$\mathrm{e}=$ margen de error $10 \%(0,10)$

$p=$ diversidad del universo ( $50 \%$ en el peor de los casos).

Las hipótesis centrales del estudio son las siguientes:

Ho: Existen altos niveles de Burnout por agotamiento emocional.

Ho: Existen altos niveles de por despersonalización.

Ho: Existen altos niveles de por falta de realización.

Ho: Los niveles medios de Burnout por agotamiento emocional son iguales para jóvenes y mayores.

Ho: Los niveles medios de Burnout por despersonalización es igual para para jóvenes y mayores.

Ho: Los niveles medios de Burnout por falta de realización personal es igual para jóvenes y mayores.

\section{RESULTADOS}

Si bien el tamaño mínimo recomendado para la muestra fue de 31 educadores, finalmente se logró concretar un total de 49 encuestas ( 45 docentes y 4 directivos). La Tabla 3 muestra las estimaciones del nivel del índice de Burnout en los docentes para cada subescala. La tabla permite apreciar que el índice más alto de Burnout ocurre para el agotamiento emocional, puesto que un 55\% se encuentra en este rango. En el caso de la despersonalización los resultados indican que la gran mayoría de los encuestados (alrededor del 70\%) no presentan ningún problema a este respecto. En relación con la realización personal en trabajo la Tabla 3 muestra que la incidencia de este problema es más bien baja.

La Fig. 1 proporciona una visión general de la simetría de la distribución de los resultados para cada subescala, reportando la dispersión de los puntos con la mediana, los percentiles 25 y 75 , y los valores máximos y mínimos. Aquí puede apreciarse que la distribución es bastante simétrica para el agotamiento emocional y la realización personal en el trabajo, debido a que la mediana se encuentra bastante al centro de cada caja. Sin embargo, la despersonalización presenta una asimetría hacia la izquierda, al mismo tiempo que existen una serie de valores atípicos (outliers) hacia la derecha.

Tabla 3: Burnout en docentes del Liceo.

\begin{tabular}{l|ccc|ccc|ccc}
\hline & \multicolumn{3}{|c|}{ Agotamiento Emocional } & \multicolumn{3}{|c|}{ Despersonalización } & \multicolumn{3}{c}{ Realización Personal } \\
\hline Nivel & Frec. & $\%$ & Cum. & Frec. & $\%$ & Cum. & Frec. & $\%$ & Cum. \\
\hline Bajo & 11 & 22.5 & 22.5 & 34 & 69.4 & 69.4 & 19 & 38.8 & 38.8 \\
Medio & 11 & 22.5 & 44.9 & 7 & 14.3 & 83.7 & 19 & 38.8 & 77.6 \\
Alto & 27 & 55.1 & 100.0 & 8 & 16.3 & 100.0 & 11 & 22.5 & 100.0 \\
\hline Total & 49 & 100.0 & & 49 & 100.0 & & 49 & 100.0 & \\
\hline
\end{tabular}

En la Tabla 4 se analiza si la edad de los docentes tiene alguna relación con los niveles de Burnout en cada una de las diferentes dimensiones. En cada celda de la Tabla 4 se presenta el número de observaciones (cantidad de encuestados), y debajo el puntaje promedio para cada componente del Burnout. En relación al agotamiento emocional, la tabla sugiere que no existen grandes diferencias en la distribución del Burnout según la edad. Lo mismo ocurre para la despersonalización y la realización personal en el trabajo. En efecto, la Fig. 2a permite visualizar con mayor claridad una pequeña disminución de los niveles de agotamiento emocional, comparando el grupo de docentes jóvenes a edades superiores a los 30 años. Pero el intervalo de confianza (en gris) indica que las diferencias no son significativas, por cuanto a los tres intervalos de confianza incluyen los valores medios de los tres grupos de edad. En el caso de la despersonalización, la Fig. $2 \mathrm{~b}$ muestra nuevamente diferencias no significativas entre grupos de edad, aunque puede apreciarse una disminución de la dispersión en el grupo de mayor edad. Finalmente, en el caso de la realización personal en el trabajo, la Fig. 2c pareciera sugerir un incremento de Burnout en el grupo de mayor edad, sin embargo, el intervalo de confianza descarta estadísticamente esta hipótesis. Para la Fig. 2 se estableció como límite exterior 2 deviaciones estándar para buscar outliers, y el límite interior se estableció a 1 deviación estándar. 
Tabla 4: Burnout por edad en docentes del Liceo

\begin{tabular}{l|ccc|ccc|ccc}
\hline & \multicolumn{3}{|c|}{ Agotamiento Emocional } & \multicolumn{3}{c|}{ Despersonalización } & \multicolumn{3}{c}{ Realización Personal } \\
\hline & $20-30$ & $31-50$ & +51 & $20-30$ & $31-50$ & +51 & $20-30$ & $31-50$ & +51 \\
& años & años & años & años & años & años & años & años & años \\
\hline Bajo & 1.0 & 7.0 & 3.0 & 13.0 & 12.0 & 9.0 & 9.0 & 8.0 & 2.0 \\
& 17.0 & 13.4 & 12.7 & 1.6 & 0.2 & 1.8 & 3.8 & 3.8 & 5.5 \\
\hline Medio & 5.0 & 2.0 & 4.0 & 2.0 & 2.0 & 3.0 & 7.0 & 4.0 & 8.0 \\
& 21.8 & 21.5 & 24.8 & 7.0 & 7.0 & 6.0 & 11.0 & 9.8 & 11.8 \\
\hline Alto & 14.0 & 8.0 & 5.0 & 5.0 & 3.0 & & 4.0 & 5.0 & 2.0 \\
& 33.6 & 33.9 & 33.0 & 15.6 & 10.7 & & 19.3 & 17.2 & 16.0 \\
\hline
\end{tabular}

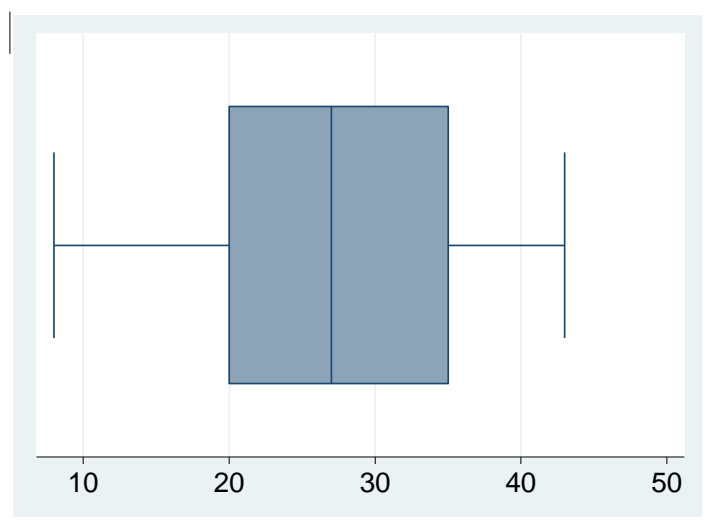

Fig. 1 a) Distribución de Puntajes: Agotamiento Emocional

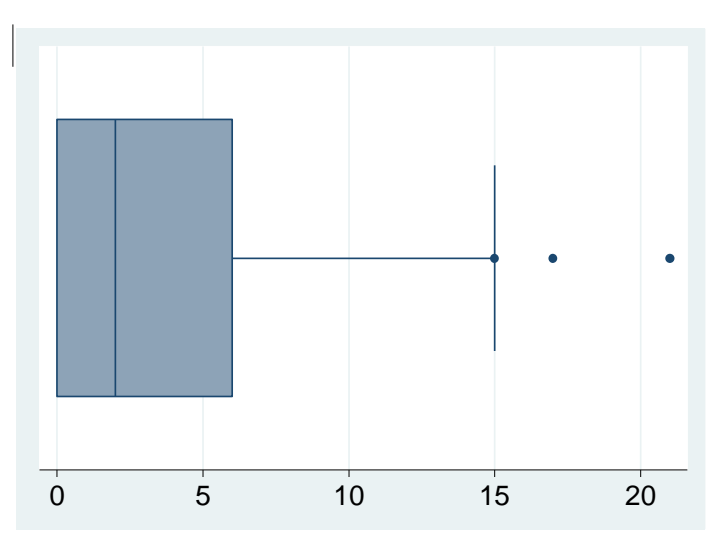

Fig. 1 b) Distribución de Puntajes: Despersonalización

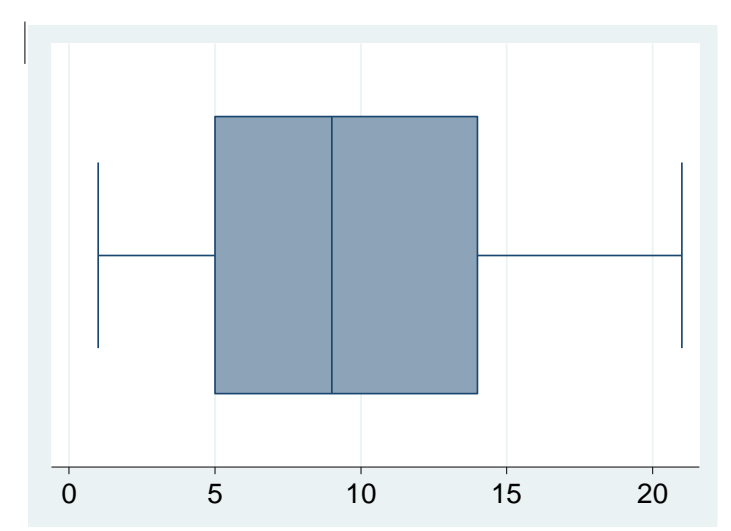

Fig. 1 c) Distribución de Puntajes: Realización Personal

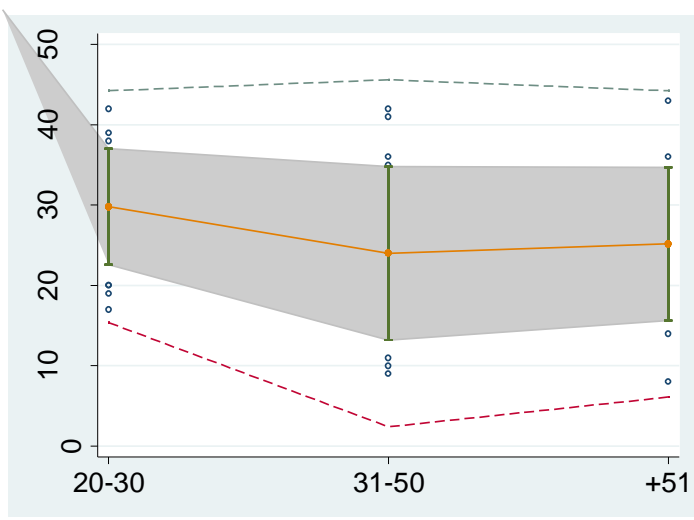

Fig. 2 a) Medias y Desv. Estandar por edad: Agotamiento Emocional

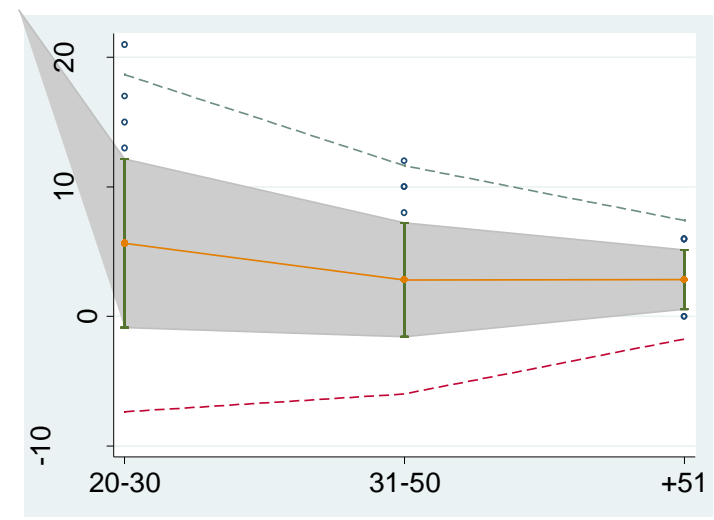

Fig. 2 b) Medias y Desv. Estandar por edad: Despersonalización

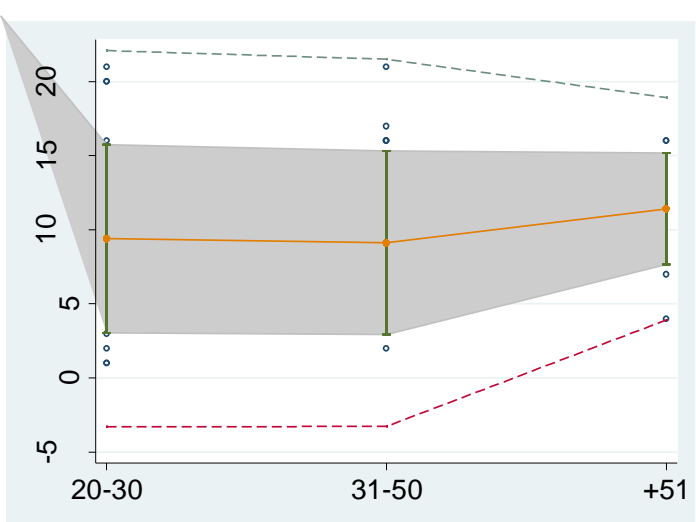

Fig. 2 c) Medias y Desv. Estandar por edad: Realización Personal 
Adicionalmente se realizaron estimaciones intentando relacionar los niveles de Burnout con otros factores socioeconómicos, tales como las horas lectivas (dictadas en aula), los años de experiencia laboral docente, y el tipo de contrato laboral. Sin embargo, en todos estos casos los resultados sugirieron la inexistencia estadística de tal relación, de modo que los resultados no son reportados aquí.

\section{DISCUSIÓN FINAL}

Los resultados del estudio sugieren que globalmente el nivel de Síndrome de Burnout es de tipo medio o bajo en los educadores del Liceo. Esto es consistente con el resultado reportado por otros estudios similares. Solamente el agotamiento emocional mostró tener una incidencia importante en los educadores encuestados, mientras que los problemas de despersonalización y de falta de realización personal en el trabajo se encuentran en niveles bajos y bajo-medio respectivamente. Esto sugiere que el mayor problema detectado se relaciona con el agotamiento, tanto físico como mental. Esto puede explicarse en el esfuerzo que implica para los educadores realizar su labor pedagógica, lo que incluye no solo impartir clases, sino también la realización de otras actividades, como la planificación y gestión administrativa. En parte, esto también puede explicarse por el hecho de que un importante número de los educadores encuestados trabajan en más de un establecimiento educacional con el objetivo de incrementar sus ingresos económicos. Los estándares de oportunidad actuales para el proyecto educativo en muchas ocasiones no alcanzan los niveles mínimos requeridos, y ello también puede originar despersonalización y falta de realización personal (Cárdenas y Hernández, 2012).

Por otro lado, la mayor parte de los docentes y directivos no presenta una sensación de despersonalización, insensibilidad, despreocupación o endurecimiento en su relación con los estudiantes. Tampoco puede observarse que consideren que su trabajo sea poco relevante, y que presenten bajo interés para trabajar con estudiantes. Es decir, los resultados sugieren que los niveles de burnout obedecen principalmente a factores ajenos al establecimiento educacional, y que una estrategia de intervención diseñada por la institución tendría una efectividad limitada.

Al relacionar los niveles de Burnout con la edad de los educadores, los resultados no sugieren cambios en el patrón global. En efecto, desde el punto de vista estadístico no se pudo encontrar una diferencia significativa en los niveles de agotamiento emocional, despersonalización y realización personal en el trabajo en los grupos de 20 a 30 años, 31 a 50 años, y los mayores de 51 años. En cuanto a la relación de Burnout y la edad, la literatura proporciona evidencias mixtas. Si bien Maslach y Jackson (1986) indican que debiera esperarse una reducción de los niveles de Burnout con la edad, la disminución que se ha detectado aquí en el caso de agotamiento emocional es baja (Fig. 2a), y no resulta ser estadísticamente significativa. Una aspecto que convendría analizar en futuros estudios es en qué medida las oportunidades de enseñar y de aprender se relaciona con los índices de burnout en los docentes (Cárdenas y Hernández, 2012).

Finalmente, los resultados de este estudio arrojan globalmente indicadores que permiten observar con optimismo el futuro de la carrera docente en los Liceos públicos regionales. Los resultados también sugieren que es valioso estudiar o plantear en un estudio futuro el análisis de la relación entre el Síndrome de Burnout y los niveles de compensación económica, las horas lectivas, por cuanto se obtuvo alguna evidencia de que existe un importante número de educadores en ejercicio, los que prestan servicios en más de un establecimiento educacional, con una extensa jornada laboral. A pesar de esto, los resultados aquí logrados son de interés tanto para los directivos del mismo Liceo, como también para los estamentos directivos de la educación pública. La aplicación de un test de este tipo de manera regular en los distintos liceos debiera permitir la detección de los síntomas del "profesor quemado", y entonces emprender acciones correctivas por parte de las autoridades, si es que resultara ser aconsejable.

\section{CONCLUSIONES}

De acuerdo a los resultados presentados y discutidos en las secciones anteriores, se puede obtener las siguientes conclusiones principales: (i) El nivel de Síndrome de Burnout es de tipo medio o bajo en los educadores del Liceo; (ii) La mayor parte de los educadores no muestran despersonalización, insensibilidad o despreocupación con los estudiantes; y (iii) La edad de los educadores no influye en los niveles de agotamiento emocional, despersonalización y realización personal.

\section{REFERENCIAS}

Báez, Y.A., M. A. Rodríguez, E.J. de la Vega y D.A. Tlapa, Factores que influyen en el error humano de los trabajadores en líneas de montaje manual. Información Tecnológica, http://dx.doi.org/10.4067/S071807642013000600010, 24(6), 67-78 (2013) 
Buzzetti, M., Validación del Maslach Burnout Inventory (MBI), en dirigentes del Colegio de Profesores A.G. de Chile. Memoria de Título de Psicóloga. Universidad de Chile, Facultad de Ciencias Sociales (2005)

Cárdenas, R. y F. Hernandez, Towards the formulation of a proposal for opportunity-to-learn standards in EFL learning and teaching. Íkala, 16 (28), 231-258 (2011)

Cárdenas, R. y F. Hernández, Estándares de oportunidad para la enseñanza y el aprendizaje: perspectivas de profesores colombianos. PROFILE, 14(2), ISSN: 1657-0790, Bogotá, Colombia, 77-94 (2012)

Colino, N. y P. Pérez, El síndrome de burnout en un grupo de profesores de enseñanza secundaria en Montevideo. Prensa Médica Latinoamericana, Ciencias Psicológicas, 9(1), 27 - 41 (2015)

Correa-Correa, Z., El Síndrome de Burnout en Profesores Universitarios de los Sectores Público y Privado. ISSN: 2250-7663 - Epidemiología y Salud, 1(2), 19-24 (2012)

Craiovan, P.M., Correlations between Perfectionism, Stress, Psychopathological Symptoms and Burnout in the Medical Field. Procedia - Social and Behavioral Sciences, 127(22), 529-533 (2014)

Fiorilli, C., P. Gabola, A. Pepe, N. Meylan, D. Curchod-Ruedi, O. Albanese, P.A. Doudin, The effect of teachers' emotional intensity and social support on burnout syndrome. A comparison between Italy and Switzerland. Revue Européenne de Psychologie Appliquée / European Review of Applied Psychology, 65(6), 275-283 (2015)

Kim, J., P. Youngs y K. Frank, Burnout contagion: Is it due to early career teachers' social networks or organizational exposure? Teaching and Teacher Education, 66, 250-260 (2017)

Košir, K., S. Tement, M. Licardo y K. Habe, Two sides of the same coin? The role of rumination and reflection in elementary school teachers' classroom stress and burnout. Teaching and Teacher Education, 47, 131-141 (2015)

Lazarus, R.S. y Folkman, S., Stress, Appraisal, and Coping. Springer Publishing Company, ISBN-10: 0826141919 (1984)

Lauermann, F. y J. König, Teachers' professional competence and wellbeing: Understanding the links between general pedagogical knowledge, self-efficacy and burnout. Learning and Instruction, 45, 9-19 (2016)

Lim, S. y S. Eo, The mediating roles of collective teacher efficacy in the relations of teachers' perceptions of school organizational climate to their burnout. Teaching and Teacher Education, 44, 138-147 (2014)

Maslach, C. Burned-out. Human Behavior, 5, 16-22, September (1976)

Maslach, C. y S.E. Jackson, Maslasch Burnout Inventory Manual. Palo Alto California: Consulting Psychology Press (1986)

Mérida-López, S. y N. Extremera, Emotional intelligence and teacher burnout: A systematic review. International Journal of Educational Research, 85, 121-130 (2017)

Mosquera, R., L. Parra-Osorio y O.D. Castrillón, Metodología para la Predicción del Grado de Riesgo Psicosocial en Docentes de Colegios Colombianos Utilizando Técnicas de Minería de Datos. Información Tecnológica, http://dx.doi.org/10.4067/S0718-07642016000600026, (27) 6, 259-272 (2016)

Muñoz, M. y P. Molina, Síndrome de Burnout en el Equipo de Salud de una Unidad de Cuidados Intensivos, Concepción. Revista Chilena de Medicina Intensiva, 28(1), 13-18 (2013)

Muñoz, G., Prevalencia del Síndrome de Burnout en el Equipo del Centro de Salud Familiar Angachilla, Valdivia 2007. Tesis de Licenciatura en Enfermería. U. Austral de Chile. Fac. de Medicina. Escuela de Enfermería (2008)

Olivares, V.E., L. Mena-Miranda, C. Jélvez, F. Macía, Validez factorial del Maslach Burnout Inventory Human Services (MBI-HSS) en profesionales chilenos. Universitas Psychologica, doi:10.11144/Javeriana.UPSY131.vfmb, 13(1), (2014)

Ortiz, M., M. Castelvi, L. Espinoza, R. Guerrero, P. Lienqueo, R. Parra y E. Villagra, Tipos de personalidad y síndrome de burnout en educadoras de párvulos en Chile. Univ. Psychol. Bogotá, Colombia, (11) 1, 229-239, ene-mar., EISSN 2011-2777 (2012) 
Panari, Ch., D. Guglielmi, S. Simbula y M. Depolo, Can an opportunity to learn at work reduce stress? A revisitation of the job demand-control model. Journal of Workplace Learning, https://doi.org/10.1108/13665621011028611, 22 (3) 166-179 (2010)

Quaas, C., Diagnóstico de Burnout y Técnicas de Afrontamiento al Estrés en Profesores Universitarios de la Quinta Región de Chile. Psicoperspectivas. Revista de la Escuela de Psicología Facultad de Filosofía y Educación, Pontificia Universidad Católica de Valparaíso, V (1), 65 - 75 (2006)

Van den Berghe, L., B. Soenens, N. Aelterman, G. Cardon, I.B. Tallir, L. Haerens, Within-person profiles of teachers' motivation to teach: Associations with need satisfaction at work, need-supportive teaching, and burnout. Psychology of Sport and Exercise, 15 (4), 407-417 (2014)

Wang, H., N.C. Hall, S. Rahimi, Self-efficacy and causal attributions in teachers: Effects on burnout, job satisfaction, illness, and quitting intentions. Teaching and Teacher Education, 47, 120-130 (2015)

Werner, D. Percepción del Burnout y Autocuidado en Psicólogos Clínicos Infantojuveniles que trabajan en Salud Pública en Santiago de Chile. Memoria de Título de Psicóloga. Universidad de Chile, Facultad de Ciencias Sociales, (2010) 
